\title{
Observation of Quantum Motion of a Nanomechanical Resonator
}

\author{
Amir H. Safavi-Naeini, Jasper Chan, Jeff T. Hill, Thiago P. Mayer Alegre, Alex Krause, and Oskar Painter* \\ Thomas J. Watson, Sr., Laboratory of Applied Physics, California Institute of Technology, Pasadena, California 91125, USA
}

(Received 14 September 2011; published 17 January 2012)

\begin{abstract}
In this Letter we use resolved sideband laser cooling to cool a mesoscopic mechanical resonator to near its quantum ground state (phonon occupancy $2.6 \pm 0.2$ ), and observe the motional sidebands generated on a second probe laser. Asymmetry in the sideband amplitudes provides a direct measure of the displacement noise power associated with quantum zero-point fluctuations of the nanomechanical resonator, and allows for an intrinsic calibration of the phonon occupation number.
\end{abstract}

DOI: 10.1103/PhysRevLett.108.033602

PACS numbers: 42.50.Wk, 42.65.- k, 62.25. $-\mathrm{g}$

Experiments with trapped ions and neutral atoms [1-3], dating back several decades, utilized techniques such as resolved sideband laser cooling and motional sideband absorption and fluorescence spectroscopy to cool and measure a single trapped particle in its vibrational quantum ground state. These experiments generated significant interest in the coherent control of motion and the quantum optics of trapped atoms and ions [4], and were important stepping stones towards the development of ion-trap based quantum computing [5,6]. Larger scale mechanical objects, such as fabricated nanomechanical resonators, have only recently been cooled close to their quantum mechanical ground state of motion [7-14]. In a pioneering experiment by O'Connell, et al. [11], a piezoelectric nanomechanical resonator has been cryogenically cooled $\left(T_{b} \sim\right.$ $25 \mathrm{mK}$ ) to its vibrational ground state and strongly coupled to a superconducting circuit qubit allowing for quantum state preparation and readout of the mechanics. An alternate line of research has been pursued in circuit and cavity optomechanics [15], where the position of a mechanical oscillator is coupled to the frequency of a high- $Q$ electromagnetic resonance allowing for backaction cooling [16,17] and continuous position readout of the oscillator. Such optomechanical resonators have long been pursued as quantum-limited sensors of weak classical forces $[9,15,18-20]$, with more recent studies exploring optomechanical systems as quantum optical memories and amplifiers [21-24], quantum nonlinear dynamical elements [25], and quantum interfaces in hybrid quantum systems [26-29].

Despite the major advances in circuit and cavity optomechanical systems made in the last few years, all experiments to date involving the cooling of mesoscopic mechanical oscillators have relied on careful measurement and calibration of the motion-induced scattering of light to obtain the average phonon occupancy of the oscillator, $\langle n\rangle$. Approach towards the quantum ground state in such experiments is manifest only as a weaker measured signal, with no evident demarcation between the classical and quantum regimes of the oscillator. A crucial aspect of zero-point fluctuations (zpfs) of the quantum ground state is that they cannot supply energy, but can only contribute to processes where energy is absorbed by the mechanics. This is different from classical noise, and techniques that attempt to measure zero-point motion without being sensitive to this aspect (i.e., standard continuous linear position detection) can always be interpreted classically and described by some effective temperature.

A more direct method of thermometry and characterization of the quantized nature of a mechanical oscillator, one particularly suited to small $\langle n\rangle$ and utilized in the abovementioned trapped atom experiments [1-3], is referred to as motional sideband spectroscopy. This method relies on the fundamental asymmetry in the quantum processes of phonon absorption from (proportional to $\langle n\rangle$ ) and emission into (proportional to $\langle n\rangle+1$ ) the mechanical oscillator. In the case of atomic systems, this asymmetry can be measured in the motionally generated Stokes and anti-Stokes sidebands in either the fluorescence or absorption spectrum of the atom. The ratio of the Stokes to anti-Stokes sideband amplitudes $[(\langle n\rangle+1) /\langle n\rangle]$ deviates significantly from unity as the quantum ground state is reached $(\langle n\rangle \rightarrow 0)$, and provides a self-calibrated reference for the phonon occupancy. In the present experiment, we cool a nanomechanical resonator to near its quantum ground state, and measure the asymmetry in the motional sidebands utilizing a form of resolved sideband spectroscopy based upon the filtering properties of a high- $Q$ optical cavity with linewidth narrower than the mechanical frequency.

The cavity optomechanical system studied in this Letter consists of a patterned silicon nanobeam which forms an optomechanical crystal (OMC) [30] capable of localizing both optical and acoustic waves (see Fig. 1). The cavity is designed to have two optical resonances, one for cooling and one for readout of mechanical motion. The cooling mode is chosen as the fundamental mode of the patterned nanobeam cavity, with a frequency $\omega_{c} / 2 \pi=205.3 \mathrm{THz}$ and a corresponding free-space wavelength of $\lambda_{c}=$ $1460 \mathrm{~nm}$. The readout mode is the second-order mode of the cavity with $\omega_{r} / 2 \pi=194.1 \mathrm{THz}\left(\lambda_{r}=1545 \mathrm{~nm}\right)$. An in-plane mechanical breathing mode at $\omega_{m} / 2 \pi=$ $3.99 \mathrm{GHz}$, confined at the center of the nanobeam due to 


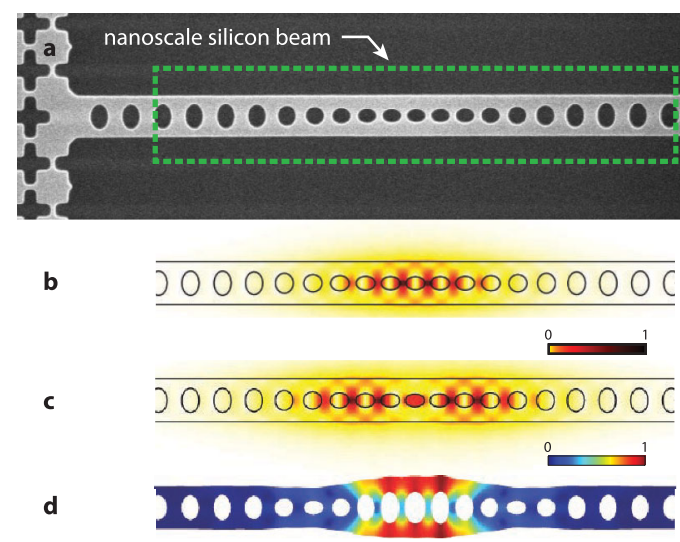

FIG. 1 (color online). (a) A scanning electron micrograph of the silicon nanobeam optomechanical cavity. Finite-element method (FEM) numerical simulations of the electric field amplitude of the (b) first- and (c) second-order optical modes of the cavity which are used for cooling and probing the mechanical motion, respectively. (d) FEM numerical simulation showing the displacement amplitude of the coupled breathing mechanical mode.

acoustic Bragg reflection, couples via radiation pressure to both optical resonances.

An illustration of the experimental apparatus used to cool and measure the $\mathrm{OMC}$ nanomechanical oscillator is shown in Fig. 2. In order to precool the oscillator, the silicon sample is mounted inside a Helium flow cryostat. For a sample mount temperature of $6.3 \mathrm{~K}$, the thermal bath temperature of the mechanical mode is measured to be $18 \mathrm{~K}$ (thermal phonon occupation of $n_{b}=94$ phonons) through optical measurements described below. At this temperature the breathing mode damping rate to the thermal bath is found to be $\gamma_{i} / 2 \pi=43 \mathrm{kHz}$. The optical resonances of the OMC cavity are measured to have total damping rates of $\kappa_{c} / 2 \pi=$ $390 \mathrm{MHz}$ and $\kappa_{r} / 2 \pi=1.0 \mathrm{GHz}$ for the cooling and readout modes, respectively. An optical fiber taper is used to evanescently couple light to and from the OMC cavity. Utilizing piezoelectric stages, the taper is positioned to the side of the nanobeam cavity and placed in contact with the surface of the silicon microchip surrounding the suspended nanobeam. In this scheme, the fiber taper runs approximately parallel to the nanobeam, and can be rigidly mounted at a prescribed nanoscale gap from the nanobeam. For the taper-to-nanobeam gap used here $(\$ 200 \mathrm{~nm})$, the coupling rate to the fiber taper waveguide is approximately $\kappa_{e, c} / 2 \pi=46 \mathrm{MHz}$ for the cooling mode and $\kappa_{e, r} / 2 \pi=$ $300 \mathrm{MHz}$ for the readout mode.

A Hamiltonian describing the coupled OMC cavity system is given by $\hat{H}=\hbar\left(\omega_{r}+g_{r} \hat{x} / x_{\mathrm{zpf}}\right) \hat{a}^{\dagger} \hat{a}+\hbar\left(\omega_{c}+\right.$ $\left.g_{c} \hat{x} / x_{\mathrm{zpf}}\right) \hat{c}^{\dagger} \hat{c}+\hbar \omega_{m} \hat{b}^{\dagger} \hat{b}$, where $\hat{c}\left(\hat{c}^{\dagger}\right)$ and $\hat{a}\left(\hat{a}^{\dagger}\right)$ are the annihilation (creation) operators for photons in the cooling and readout modes, respectively, and $\hat{x} \equiv x_{\mathrm{zpf}}\left(\hat{b}^{\dagger}+\hat{b}\right)$ is the displacement operator of the breathing mode with $\hat{b}^{\dagger}$ $(\hat{b})$ the phonon creation (annihilation) operator. $x_{\mathrm{zpf}}$, the

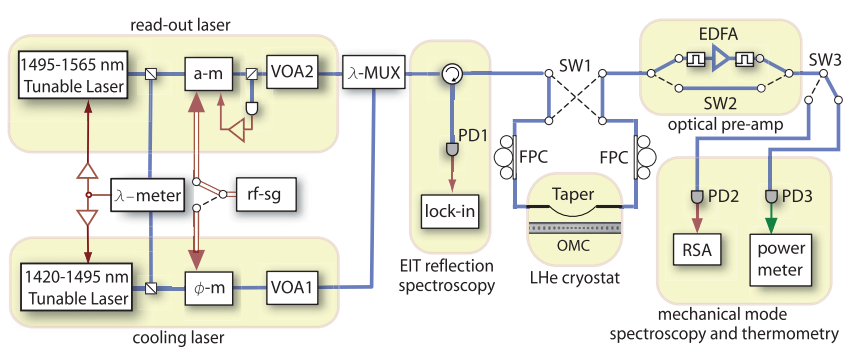

FIG. 2 (color online). Schematic of the experimental set-up. Two narrowband lasers (linewidth $\sim 300 \mathrm{kHz}$ ) are used to independently cool and readout the motion of the breathing mechanical mode of the OMC cavity. The $1500 \mathrm{~nm}$ (readout) and $1400 \mathrm{~nm}$ (cooling) laser beams are passed through variable optical attenuators (VOAs) to set the laser power, and combined at a wavelength multiplexer ( $\lambda$-MUX) before being sent into the cryostat through an optical fiber. Transmission of the $1500 \mathrm{~nm}$ readout beam through the OMC cavity, collected at the output end of the optical fiber, is filtered from the $1400 \mathrm{~nm}$ cooling beam light via a bandpass filter, preamplified by an Erbiumdoped fiber amplifier (EDFA), and detected on a high-speed photodetector (PD2) connected to a real-time spectrum analyzer (RSA). An optical wave meter ( $\lambda$-meter) is used to monitor both the cooling and readout laser frequencies. The optical reflection from the cavity is used to perform EIT-like spectroscopy [22] on both the readout and cooling cavity modes. Other components are: amplitude-modulation (a-m) and phase-modulation $(\phi-\mathrm{m})$ electro-optic modulators, fiber polarization controller (FPC), swept frequency radio-frequency signal generator (rf-sg), lockin amplifier (lock-in), and optical switches (SW).

mode's zero-point fluctuation amplitude, is estimated to be $2.7 \mathrm{fm}$ from FEM simulations. The zero-point optomechanical coupling rates are determined from measurements of the optically-induced damping of the mechanical mode [13] to be $g_{c} / 2 \pi=960 \mathrm{kHz}$ and $g_{r} / 2 \pi=430 \mathrm{kHz}$ for the cooling and readout modes, respectively.

As alluded to above, resolved sideband cooling in optomechanical cavities follows physics which is formally similar to the Raman processes used to cool ions to their motional ground state [1]. A cooling laser, with frequency $\omega_{l}=\omega_{c}-\omega_{m}$, is tuned a mechanical frequency below that of the cooling cavity resonance of the OMC, giving rise to an intracavity photon population $n_{c}$. Motion of the mechanical oscillator causes scattering of the intracavity cooling beam laser light into Stokes and anti-Stokes sidebands at $\omega_{c}-2 \omega_{m}$ and $\omega_{c}$, respectively. Since the antiStokes sideband is resonant with the cavity at $\omega_{c}$, and $\kappa_{c}<\omega_{m}$, the anti-Stokes optical up-conversion process is greatly enhanced relative to the Stokes down-conversion process, leading to cooling of the mechanical mode. Assuming a deeply resolved sideband system $\left(\kappa_{c} / \omega_{m} \ll 1\right)$, the backaction cooled mechanical mode occupancy is approximately given by $\langle n\rangle_{c}=$ $\gamma_{i} n_{b} /\left(\gamma_{i}+\gamma_{c}\right)[16,17]$.

Optical scattering of the intracavity light field can also be used to read out the motion of the coupled mechanical 
oscillator. For a quantum harmonic oscillator, the noise power spectral density (PSD) of the oscillator's position is equal to [20],

$$
\begin{aligned}
S_{x x}(\omega) / x_{\mathrm{zpf}}^{2}= & \frac{\gamma\langle n\rangle}{\left(\omega_{m}+\omega\right)^{2}+(\gamma / 2)^{2}} \\
& +\frac{\gamma(\langle n\rangle+1)}{\left(\omega_{m}-\omega\right)^{2}+(\gamma / 2)^{2}},
\end{aligned}
$$

where $\gamma$ is the total mechanical damping rate. The asymmetric zero-point motion contribution to $S_{x x}(\omega)$ [illustrated in Fig. 3(a)] arises from the noncommutivity of position and momentum operators in quantum mechanics. This absorption-emission asymmetry has no classical analogue; of course, at high phonon occupation numbers where $\langle n\rangle \approx\langle n\rangle+1$, the classically symmetric spectral density is recovered. Since the optical cavity frequency is linearly coupled to the position of the mechanical oscillator, the displacement noise spectrum is imprinted on the photons leaving the cavity and can be measured optically.

Specifically, consider a readout laser with frequency $\omega_{l r}$ and detuning $\Delta \equiv \omega_{r}-\omega_{l r}$ from the readout cavity mode. The optical power spectrum of the motional sidebands of the transmitted readout beam leaving the cavity is given by [16],

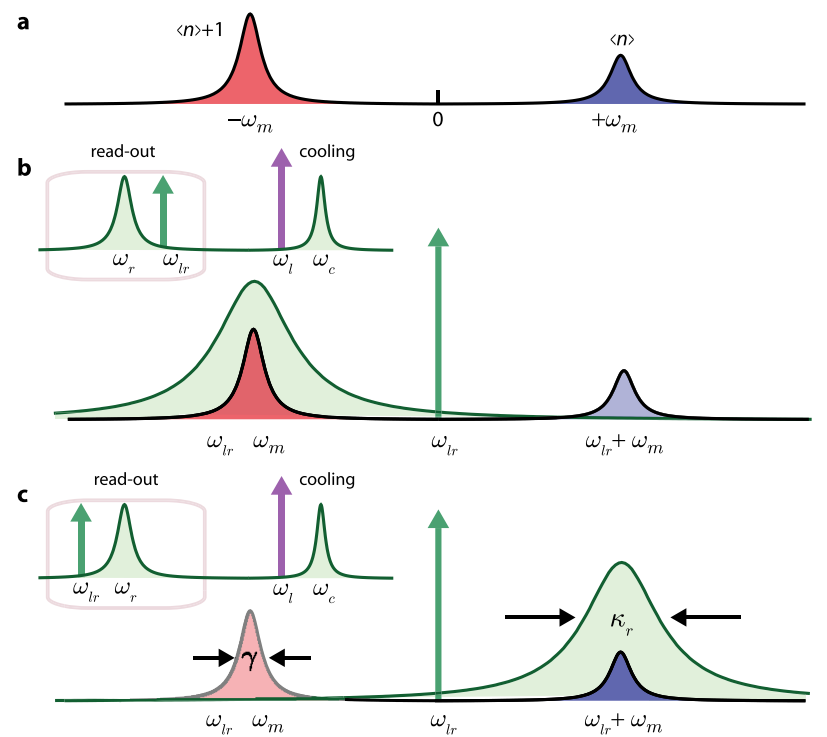

FIG. 3 (color online). (a) Displacement noise PSD, $S_{x x}$, of a quantum simple harmonic oscillator, plotted against $-\omega$ for clarity. (b) Scheme for measurement of the down-converted (Stokes) motional sideband. Here the readout laser (vertical arrow; frequency $\omega_{l r}$ ) is detuned a mechanical frequency above that of the readout cavity resonance (broad solid curve). (c) Corresponding scheme for measurement of the up-converted (anti-Stokes) motional sideband. The linewidth of the readout cavity $\left(\kappa_{r}\right)$ and the mechanical resonance $(\gamma)$ are indicated. Insets to (b) and (c) show a zoomedout spectra indicating the relative frequency of the cooling cavity mode and cooling laser.

$$
\begin{aligned}
S\left(\omega+\omega_{l r}\right)= & \frac{\kappa_{e, r}}{2 \pi \kappa_{r}} \frac{A_{-}^{(r)} \gamma\langle n\rangle}{\left(\omega_{m}-\omega\right)^{2}+(\gamma / 2)^{2}} \\
& +\frac{\kappa_{e, r}}{2 \pi \kappa_{r}} \frac{A_{+}^{(r)} \gamma(\langle n\rangle+1)}{\left(\omega_{m}+\omega\right)^{2}+(\gamma / 2)^{2}} .
\end{aligned}
$$

Here $A_{+}^{(r)}$ and $A_{-}^{(r)}$ are the detuning-dependent anti-Stokes and Stokes motional scattering rates, respectively, of the readout laser, given by $A_{ \pm}^{(r)}=g_{r}^{2} \kappa_{r} n_{r} /\left[\left(\Delta \pm \omega_{m}\right)^{2}+\right.$ $\left.\left(\kappa_{r} / 2\right)^{2}\right]$.

As illustrated in Fig. 3(b) and 3(c), the optical readout cavity can be used to selectively filter the positive or negative frequency components of $S(\omega)$. For a detuning $\Delta=-\omega_{m}$ for the readout laser, $A_{+}^{(r)} \gg A_{-}^{(r)}$, resulting in a Lorentzian signal with area $I_{-}$proportional to $\langle n\rangle+1$. Conversely, a detuning of $\Delta=\omega_{m}$ results in $A_{-}^{(r)} \gg A_{+}^{(r)}$, producing a signal of area $I_{+}$proportional to $\langle n\rangle$. Comparison of the area under the Lorentzian part of the measured photocurrent PSD of the transmitted readout laser for detunings $\Delta= \pm \omega_{m}$, can then be used to infer the mechanical mode occupancy,

$$
\eta \equiv I_{-} / I_{+}-1=\frac{1}{\langle n\rangle} .
$$

This simple argument neglects the backaction of the readout beam on the mechanical oscillator. In particular, the mechanical damping rate becomes detuning dependent, with $\gamma_{ \pm} \equiv\left(\gamma_{i}+\gamma_{c}\right)\left(1 \pm C_{r}\right)$ for $\Delta= \pm \omega_{m}$. Here $C_{r} \equiv$ $\left|A_{+}^{(r)}-A_{-}^{(r)}\right| /\left(\gamma_{i}+\gamma_{c}\right)$ is the effective cooperativity of the readout beam in the presence of the strong cooling beam, and can be found from the measured spectra by the relation, $C_{r}=\left(\gamma_{+}-\gamma_{-}\right) /\left(\gamma_{+}+\gamma_{-}\right)$. The backaction of the readout beam also results in a corresponding change in the phonon occupancy, given by $\langle n\rangle_{ \pm}=\langle n\rangle_{c} /\left(1 \pm C_{r}\right)$ for $\Delta= \pm \omega_{m}$. Here $\langle n\rangle_{c}$ is the mechanical mode occupancy due to backaction from the cooling beam only. Adding in a correction for the readout laser backaction, one finds the following relation between the measured motional sidebands and the phonon occupancy of the cooled mechanical oscillator,

$$
\eta^{\prime} \equiv \frac{I_{-} / I_{+}}{1+C_{r}}-\frac{1}{1-C_{r}}=\frac{1}{\langle n\rangle_{c}},
$$

where for $C_{r} \ll 1$, we recover the standard relation given in Eq. (3).

Figure 4 summarizes the measurement results of the calibrated mechanical mode thermometry and motional sideband asymmetry for the silicon OMC cavity. These measurements are performed with the cooling laser locked a mechanical frequency to the red of the fundamental mode of the OMC cavity, and the cooling laser power swept from $n_{c} \sim 1$ to 330 (maximum input power of $250 \mu \mathrm{W}$ ). A much weaker readout laser $\left(C_{r} \ll 1\right)$ is used to both estimate the mechanical mode phonon occupancy and to compare the motional sideband amplitudes. Locking of the 

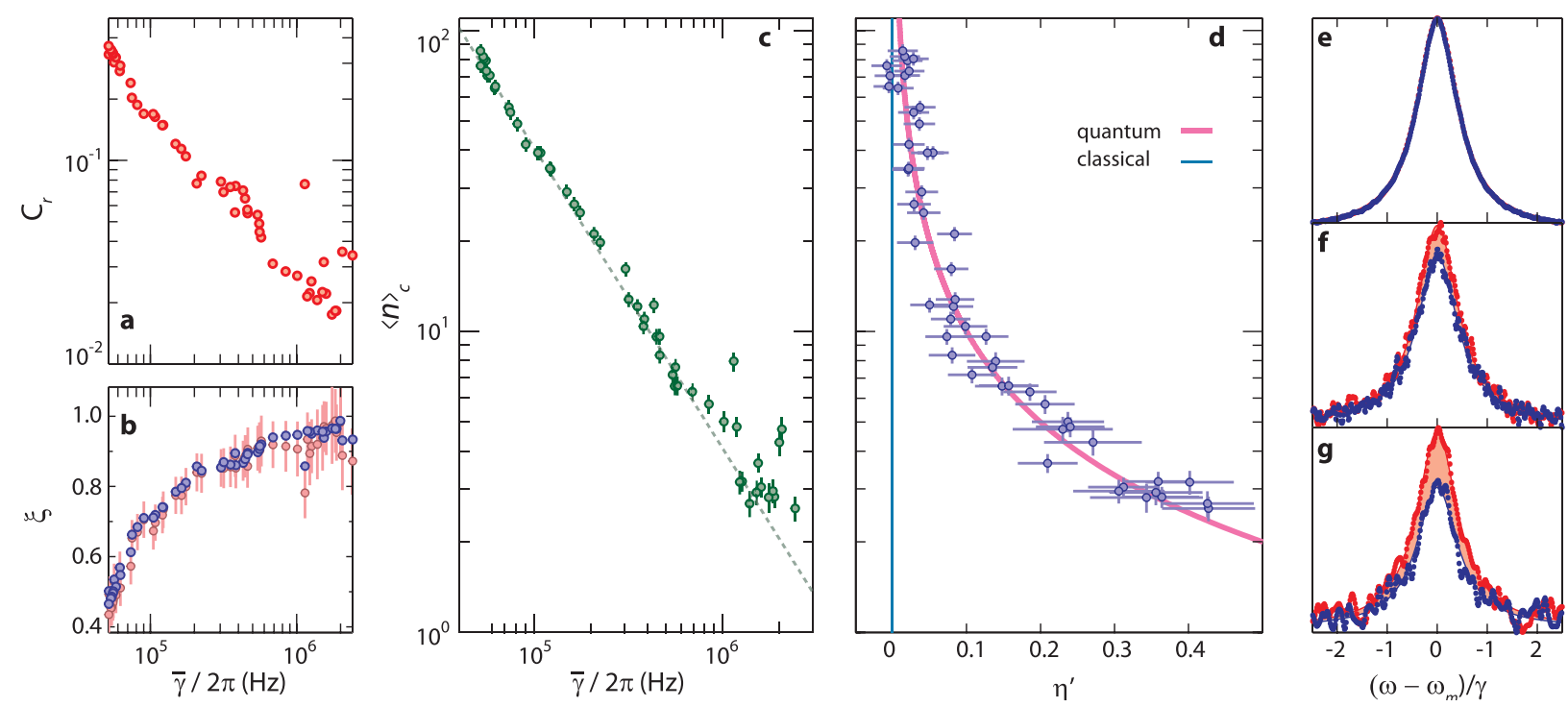

FIG. 4 (color). (a) Plot of the cooperativity of the readout beam as a function of damped mechanical linewidth. (b) Plot of the measured ratios $\gamma_{-} / \gamma_{+}$(blue $\bigcirc$ ) and $\langle n\rangle_{+} /\langle n\rangle_{-}$(pink $\bigcirc$ ). (c) Plot of the mechanical mode phonon occupancy, $\langle n\rangle_{c}$, as a function of the optically damped mechanical linewidth, $\bar{\gamma}$. The dashed line is the predicted phonon number $\gamma_{i} n_{b} / \bar{\gamma}$ from an ideal backaction cooling model. Vertical error bars in (b) and (c) indicate uncertainty in the calibrated phonon occupancy due to uncertainty in the system parameters and a 95\% confidence interval on the Lorentzian fits to spectra. (d) Plot of the asymmetry $\left(\eta^{\prime}\right)$ in the measured Stokes and anti-Stokes sidebands of the readout laser for each calibrated measurement of $\langle n\rangle_{c}$. The horizontal error bars arise from a $2 \%$ uncertainty in the transmitted readout laser beam power between detunings $\Delta= \pm \omega_{m}$, and a 95\% confidence interval in the Lorentzian fits to the measured spectra. The vertical error bars in $\langle n\rangle_{c}$ are the same as in (c). The classical (blue curve) and quantum mechanical (pink curve) relations for the sideband asymmetry are also plotted. (e)- $(\mathrm{g})$ Plot of the measured Stokes (red curve) and anti-Stokes (blue curve) readout beam spectra for (from top to bottom) $\langle n\rangle_{c}=85,6.3$, and 3.2 phonons. For clarity, we have divided out the readout backaction from each spectra by multiplying the measured spectra at detunings $\Delta= \pm \omega_{m}$ by $\gamma_{ \pm}$. Additionally, we have plotted the horizontal axis in units of $\gamma$, and rescaled the vertical axis for different $\langle n\rangle_{c}$ to keep the areas directly comparable. The difference in the Stokes and anti-Stokes spectra, which arises due to the quantum zero-point fluctuation of the mechanical system, is shown as a shaded region.

cooling and readout lasers utilizes a high resolution wave meter (10 MHz resolution) to set the absolute laser frequency and a weak probe beam to determine the lasercavity detuning. Here the weak probe beam is generated from the cooling or readout laser via electro-optic modulation, similar to the electromagnetically induced transparency (EIT) spectroscopy described in Ref. [22]. The laser-cavity lock for both cooling and readout lasers is performed every few minutes, multiplexed in time between measurements of the phonon occupancy. With the readout laser set to a detuning $\Delta=\omega_{m}$ from the readout cavity, a Lorentzian spectrum with linewidth $\gamma_{+}$and integrated area $I_{+}$is measured in the readout laser photocurrent PSD, from which a mode occupancy of $\langle n\rangle_{+}$is inferred from a careful calibration of the optomechanical cavity and photodetection system parameters [13]. Similarly, by placing the readout laser at $\Delta=-\omega_{m}$ we obtain spectra with linewidth $\gamma_{-}$and integrated area $I_{-} \propto\langle n\rangle_{-}+1$, from which we estimate $\langle n\rangle_{-}$.

Figure 4(a) plots the readout cooperativity $C_{r}$, calculated from the measured $\gamma_{ \pm}$, versus the mechanical damping rate $\bar{\gamma}=\left(\gamma_{+}+\gamma_{-}\right) / 2$. The ratios $\xi \equiv \gamma_{-} / \gamma_{+}$and $\langle n\rangle_{+} /\langle n\rangle_{-}$are plotted in Fig. 4(b). From $\langle n\rangle_{ \pm}$, the laser cooled phonon occupation number, $\langle n\rangle_{c}$, is calculated and plotted in Fig. 4(c) versus $\bar{\gamma}$. As expected, $\langle n\rangle_{c}$ drops approximately linearly with $\bar{\gamma}$, reaching a minimum value of approximately $2.6 \pm 0.2$ phonons. Further cooling below a single phonon has been achieved in similar devices [13]; however, in this case cooling is limited by the available power of the $1400 \mathrm{~nm}$ cooling laser. Also evident in Fig. 4(c), at the higher cooling powers, is an increased scatter and deviation of $\langle n\rangle_{c}$ from the ideal cooling curve (dashed curve). This can be attributed to optical absorption in the silicon nanobeam [13], which in this case produces a power-dependent variation in $n_{b}$ and $\gamma_{i}$ due to both the readout and cooling laser beams.

In Fig. 4(d), the measured values of the expression $\eta^{\prime}$ are plotted versus the calibrated value of $\langle n\rangle_{c}$. Also plotted are the classical and quantum values of this expression, 0 and $1 /\langle n\rangle_{c}$, respectively. A clear divergence from the classical result of $\eta^{\prime}=0$ is apparent, agreeing with the deviation due to zero-point fluctuations of the mechanical oscillator. This deviation is directly apparent in the measured spectra, shown for $\langle n\rangle_{c}=85,6.3$, and 3.2 phonons in Fig. 4(e) $-4(\mathrm{~g})$, with the shaded region corresponding to the noise power contribution due to zero-point motion. At 
the largest powers, we measure asymmetry in the motional sideband amplitudes of $\sim 40 \%$ in agreement with the inferred $\langle n\rangle_{c}=2.6$ phonons from calibrated thermometry.

While the quantum nature of a mechanical resonator will come as little surprise to most physicists, its observation through the zero-point motion is a significant step towards observing and controlling the quantum dynamics of mesoscopic mechanical systems. By demonstrating the fundamentally quantum behavior of an engineered mechanical nanostructure, we have shown that realizable optomechanical systems have the sensitivity and environmental isolation required for such quantum mechanical investigations.

The authors would like to thank Aash Clerk, Markus Aspelmeyer, and Simon Gröblacher for their valuable input at various stages in this experiment. This work was supported by the DARPA/MTO ORCHID program through a grant from AFOSR, and the Kavli Nanoscience Institute at Caltech. JC and ASN gratefully acknowledge support from NSERC .

*opainter@caltech.edu; http://copilot.caltech.edu

[1] F. Diedrich, J. C. Bergquist, W. M. Itano, and D. J. Wineland, Phys. Rev. Lett. 62, 403 (1989).

[2] P. S. Jessen, C. Gerz, P. D. Lett, W. D. Phillips, S. L. Rolston, R. J.C. Spreeuw, and C.I. Westbrook, Phys. Rev. Lett. 69, 49 (1992).

[3] C. Monroe, D. M. Meekhof, B.E. King, S. R. Jefferts, W. M. Itano, D. J. Wineland, and P. Gould, Phys. Rev. Lett. 75, 4011 (1995).

[4] C. A. Blockley, D. F. Walls, and H. Risken, Europhys. Lett. 17, 509 (1992).

[5] J. I. Cirac and P. Zoller, Phys. Rev. Lett. 74, 4091 (1995).

[6] A. Steane, Appl. Phys. B 64, 623 (1997).

[7] S. Gröblacher, J. B. Hertzberg, M. R. Vanner, S. Gigan, K. C. Schwab, and M. Aspelmeyer, Nature Phys. 5, 485 (2009).

[8] Y.-S. Park and H. Wang, Nature Phys. 5, 489 (2009).

[9] A. Schliesser, O. Arcizet, R. Rivière, G. Anetsberger, and T. J. Kippenberg, Nature Phys. 5, 509 (2009).

[10] T. Rocheleau, T. Ndukum, C. Macklin, J. B. Hertzberg, A. A. Clerk, and K. C. Schwab, Nature (London) 463, 72 (2009).
[11] A. D. O'Connell, M. Hofheinz, M. Ansmann, R. C. Bialczak, M. Lenander, E. Lucero, M. Neeley, D. Sank, H. Wang, and M. Weides et al., Nature (London) 464, 697 (2010).

[12] J. D. Teufel, T. Donner, D. Li, J. H. Harlow, M. S. Allman, K. Cicak, A. J. Sirois, J. D. Whittaker, K. W. Lehnert, and R. W. Simmonds, Nature (London) 475, 359 (2011).

[13] J. Chan, T. P. Mayer Alegre, A. H. Safavi-Naeini, J. T. Hill, A. Krause, S. Groeblacher, M. Aspelmeyer, and O. Painter, Nature (London) 478, 89 (2011).

[14] E. Verhagen, S. Deléglise, S. Weis, A. Schliesser, and T. J. Kippenberg, arXiv:1107.3761.

[15] V.B. Braginsky and A.B. Manukin, Measurements of Weak Forces in Physics Experiments (The University of Chicago Press, Chicago, IL, 1977).

[16] I. Wilson-Rae, N. Nooshi, W. Zwerger, and T. J. Kippenberg, Phys. Rev. Lett. 99, 093901 (2007).

[17] F. Marquardt, J. P. Chen, A. A. Clerk, and S. M. Girvin, Phys. Rev. Lett. 99, 093902 (2007).

[18] C. Caves, K. Thorne, R. Drever, V. D. Sandberg, and M. Zimmermann, Rev. Mod. Phys. 52, 341 (1980).

[19] C. A. Regal, J. D. Teufel, and K. W. Lehnert, Nature Phys. 4, 555 (2008).

[20] A. A. Clerk, M. H. Devoret, S. M. Girvin, F. Marquardt, and R. J. Schoelkopf, Rev. Mod. Phys. 82, 1155 (2010).

[21] D. Chang, A. H. Safavi-Naeini, M. Hafezi, and O. Painter, New J. Phys. 13, 023003 (2011).

[22] A. H. Safavi-Naeini, T.P. M. Alegre, J. Chan, M. Eichenfield, M. Winger, Q. Lin, J. T. Hill, D. Chang, and O. Painter, Nature (London) 472, 69 (2011).

[23] D. W. C. Brooks, T. Botter, N. Brahms, T. Purdy, S. Schreppler, and D. Stamper-Kurn, arXiv:1107.5609.

[24] F. Massel, T. T. Heikkilä, J.-M. Pirkkalainen, S. U. Cho, H. Saloniemi, P. Hakonen, and M. A. Sillanpää, arXiv:1107.4903.

[25] M. Ludwig, B. Kubala, and F. Marquardt, New J. Phys. 10, 095013 (2008).

[26] M. Wallquist, K. Hammerer, P. Rabl, M. Lukin, and P. Zoller, Phys. Scr. T137, 014001 (2009).

[27] K. Stannigel, P. Rabl, A. S. Sørensen, P. Zoller, and M. D. Lukin, Phys. Rev. Lett. 105, 220501 (2010).

[28] A. H. Safavi-Naeini and O. Painter, New J. Phys. 13, 013017 (2011).

[29] S. Camerer, M. Korppi, A. Jöckel, D. Hunger, T. W. Hänsch, and P. Treutlein, Phys. Rev. Lett. 107, 223001 (2011).

[30] M. Eichenfield, J. Chan, R. M. Camacho, K. J. Vahala, and O. Painter, Nature (London) 462, 78 (2009). 